\title{
A study of Riemann-Liouville fractional nonlocal integral boundary value problems
}

\author{
Bashir Ahmad ${ }^{1 *}$, Ahmed Alsaedi $^{1}$, Afrah Assolami and Ravi P Agarwal ${ }^{1,2}$
}

"Correspondence:

bashir_qau@yahoo.com

'Department of Mathematics,

Faculty of Science, King Abdulaziz

University, P.O. Box 80203, Jeddah,

21589, Saudi Arabia

Full list of author information is

available at the end of the article

\begin{abstract}
In this paper, we discuss the existence and uniqueness of solutions for a Riemann-Liouville type fractional differential equation with nonlocal four-point Riemann-Liouville fractional-integral boundary conditions by means of classical fixed point theorems. An illustration of main results is also presented with the aid of some examples.
\end{abstract}

MSC: $34 \mathrm{~A} 08 ; 34 \mathrm{~B} 10 ; 34 \mathrm{~B} 15$

Keywords: fractional differential equations; nonlocal fractional-integral boundary conditions; existence; fixed point

\section{Introduction}

In recent years, boundary value problems of nonlinear fractional differential equations with a variety of boundary conditions have been investigated by many researchers. Fractional differential equations appear naturally in various fields of science and engineering and constitute an important field of research [1-4]. As a matter of fact, fractional derivatives provide an excellent tool for the description of memory and hereditary properties of various materials and processes. This is one of the characteristics of fractional-order differential operators that contributes to the popularity of the subject and has motivated many researchers and modelers to shift their focus from classical models to fractional order models. In consequence, there has been a significant progress in the theoretical analysis like periodicity, asymptotic behavior and numerical methods for fractional differential equations. Some recent work on the topic can be found in [5-20] and the references therein.

Fractional boundary conditions (FBC) involving fractional derivative $D^{\alpha}$ of order $\alpha \epsilon$ $(0,1)$ describe an intermediate boundary between the perfect electric conductor (PEC) and the perfect magnetic conductor (PMC), whereas $\alpha=0$ and $\alpha=1$ in FBC correspond to PEC and PMC, respectively. Fractional boundary conditions (FBC) are also matched with impedance boundary conditions (IBC) in the sense that the fractional order $\alpha=0$ and $\alpha=1$ in FBC correspond to the value of impedance $Z=0$ and $Z=i \infty$. Recall that the value of the impedance $Z$ varies from 0 for PEC to $i \infty$ for PMC. For more details, see [21]. In [22], the authors recently studied a problem of Riemann-Liouville fractional differential equations with fractional boundary conditions:

$$
\begin{aligned}
& D^{\alpha} u(t)=f(t, u(t)), \quad t \in[0, T], \alpha \in(1,2], \\
& D^{\alpha-2} u\left(0^{+}\right)=b_{0} D^{\alpha-2} u\left(T^{-}\right), \quad D^{\alpha-1} u\left(0^{+}\right)=b_{1} D^{\alpha-1} u\left(T^{-}\right),
\end{aligned}
$$

○2013 Ahmad et al.; licensee Springer. This is an Open Access article distributed under the terms of the Creative Commons Attribution License (http://creativecommons.org/licenses/by/2.0), which permits unrestricted use, distribution, and reproduction in any medium, provided the original work is properly cited. 
where $D^{\alpha}$ denotes the Riemann-Liouville fractional derivative of order $\alpha$ and $b_{0} \neq 1$ and $b_{1} \neq 1$.

In this paper, motivated by [22], we study a fully Riemann-Liouville fractional nonlocal integral boundary value problem given by

$$
\left\{\begin{array}{l}
D^{\alpha} u(t)=f(t, u(t)), \quad 1<\alpha \leq 2, t \in[0, T], \\
D^{\alpha-1} u\left(0^{+}\right)-a D^{\alpha-1} u\left(T^{-}\right)=A I^{\beta} u(\xi), \\
D^{\alpha-2} u\left(0^{+}\right)-b D^{\alpha-2} u\left(T^{-}\right)=B I^{\beta} u(\eta), \quad \beta>0 ; \xi, \eta \in(0, T),
\end{array}\right.
$$

where $D^{\alpha}$ denotes the Riemann-Liouville fractional derivative of order $\alpha, f$ is a given continuous function, $I^{\beta}$ denotes the Riemann-Liouville integral of order $\beta$, and $a, A, b$, and $B$ are real constants.

The paper is organized as follows. In Section 2, we establish an auxiliary lemma which is needed to define the solutions of the given problem. Section 3 contains main results. In Section 4, we discuss some examples for the illustration of the main results.

\section{Preliminaries}

Let us recall some basic definitions of fractional theory.

Definition 2.1 The Riemann-Liouville fractional integral of order $\alpha$ for a continuous function $g:[0, \infty) \rightarrow \mathbb{R}$ is defined as

$$
I^{\alpha} g(t)=\frac{1}{\Gamma(\alpha)} \int_{0}^{t} \frac{g(s)}{(t-s)^{1-\alpha}} d s, \quad \alpha>0,
$$

provided the integral exists.

Definition 2.2 For a continuous function $g:[0, \infty) \rightarrow \mathbb{R}$, the Riemann-Liouville derivative of fractional order $\alpha>0$ is defined as

$$
D^{\alpha} g(t)=\frac{1}{\Gamma(n-\alpha)}\left(\frac{d}{d t}\right)^{n} \int_{0}^{t}(t-s)^{n-\alpha-1} g(s) d s=\left(\frac{d}{d t}\right)^{n} I^{n-\alpha} g(t), \quad n-1<\alpha \leq n,
$$

$n=[\alpha]+1$, where $[\alpha]$ denotes the integer part of the real number $\alpha$.

Lemma 2.1 For $1<\alpha \leq 2$, the solution of $D^{\alpha} u(t)=\sigma(t), t \in[0, T]$ subject to the boundary conditions given by (1.1) is

$$
\begin{aligned}
u(t)= & I^{\alpha} \sigma(t)+\left(\delta_{1} t^{\alpha-1}-\delta_{4} t^{\alpha-2}\right)\left[A I^{\alpha+\beta} \sigma(\xi)+a I^{1} \sigma(T)\right] \\
& +\left(\delta_{2} t^{\alpha-1}+\delta_{3} t^{\alpha-2}\right)\left[B I^{\alpha+\beta} \sigma(\eta)+b I^{2} \sigma(T)\right],
\end{aligned}
$$

where

$$
\begin{aligned}
\delta_{1}= & \frac{\Gamma(\alpha-1)}{\delta}\left(1-b-\frac{B \eta^{\alpha+\beta-2}}{\Gamma(\alpha+\beta-1)}\right) ; \quad \delta_{2}=\frac{A \Gamma(\alpha-1) \xi^{\alpha+\beta-2}}{\delta \Gamma(\alpha+\beta-1)} ; \\
\delta_{3}= & \frac{\Gamma(\alpha)}{\delta}\left(1-a-\frac{A \xi^{\alpha+\beta-1}}{\Gamma(\alpha+\beta)}\right) ; \quad \delta_{4}=\frac{\Gamma(\alpha)}{\delta}\left(b T-\frac{B \eta^{\alpha+\beta-1}}{\Gamma(\alpha+\beta)}\right) ; \\
\delta= & \Gamma(\alpha) \Gamma(\alpha-1)\left(1-a-\frac{A \xi^{\alpha+\beta-1}}{\Gamma(\alpha+\beta)}\right)\left(1-b-\frac{B \eta^{\alpha+\beta-2}}{\Gamma(\alpha+\beta-1)}\right) \\
& -\frac{A \Gamma(\alpha) \Gamma(\alpha-1) \xi^{\alpha+\beta-2}}{\Gamma(\alpha+\beta) \Gamma(\alpha+\beta-1)}\left(b T \Gamma(\alpha+\beta)+B \eta^{\alpha+\beta-1}\right) .
\end{aligned}
$$


Proof For arbitrary constants $c_{1}, c_{2} \in \mathbb{R}$, it is well known that the general solution of the equation $D^{\alpha} u(t)=\sigma(t), 1<\alpha \leq 2$, can be written as

$$
u(t)=c_{1} t^{\alpha-1}+c_{2} t^{\alpha-2}+I^{\alpha} \sigma(t) .
$$

From (2.3), we have

$$
\begin{aligned}
& D^{\alpha-1} u(t)=c_{1} \Gamma(\alpha)+I^{1} \sigma(t), \quad D^{\alpha-2} u(t)=c_{1} \Gamma(\alpha) t+c_{2} \Gamma(\alpha-1)+I^{2} \sigma(t), \\
& I^{\beta} u(\varrho)=\frac{c_{1} \varrho^{\alpha+\beta-1} \Gamma(\alpha)}{\Gamma(\alpha+\beta)}+\frac{c_{2} \varrho^{\alpha+\beta-2} \Gamma(\alpha-1)}{\Gamma(\alpha+\beta-1)}+I^{\alpha+\beta} \sigma(\varrho),
\end{aligned}
$$

where $\varrho$ denotes $\xi$ or $\eta$. Applying the given boundary conditions, we get

$$
\left\{\begin{array}{l}
\left(\Gamma(\alpha)(1-a)-\frac{\Gamma(\alpha) A \xi^{\alpha+\beta-1}}{\Gamma(\alpha+\beta)}\right) c_{1}-\frac{A \Gamma(\alpha-1) \xi^{\alpha+\beta-2}}{\Gamma(\alpha+\beta-1)} c_{2}=A I^{\alpha+\beta} \sigma(\xi)+a I^{1} \sigma(T), \\
-\Gamma(\alpha)\left(b T+\frac{B \eta^{\alpha+\beta-1}}{\Gamma(\alpha+\beta)}\right) c_{1}+\Gamma(\alpha-1)\left(1-b-\frac{B \eta^{\alpha+\beta-2}}{\Gamma(\alpha+\beta-1)}\right) c_{2}=B I^{\alpha+\beta} \sigma(\eta)+b I^{2} \sigma(T) .
\end{array}\right.
$$

Solving the system of equations (2.6) for $c_{1}, c_{2}$, we find that

$$
\begin{aligned}
& c_{1}=\delta_{1}\left(A I^{\alpha+\beta} \sigma(\xi)+a I^{1} \sigma(T)\right)+\delta_{2}\left(B I^{\alpha+\beta} \sigma(\eta)+b I^{2} \sigma(T)\right), \\
& c_{2}=\delta_{3}\left(B I^{\alpha+\beta} \sigma(\eta)+b I^{2} \sigma(T)\right)-\delta_{4}\left(A I^{\alpha+\beta} \sigma(\xi)+a I^{1} \sigma(T)\right) .
\end{aligned}
$$

Substituting these values in (2.3), we get

$$
\begin{aligned}
u(t)= & I^{\alpha} \sigma(t)+A\left(\delta_{1} t^{\alpha-1}-\delta_{4} t^{\alpha-2}\right) I^{\alpha+\beta} \sigma(\xi)+B\left(\delta_{2} t^{\alpha-1}+\delta_{3} t^{\alpha-2}\right) I^{\alpha+\beta} \sigma(\eta) \\
& +b\left(\delta_{2} t^{\alpha-1}+\delta_{3} t^{\alpha-2}\right) I^{2} \sigma(T)+a\left(\delta_{1} t^{\alpha-1}-\delta_{4} t^{\alpha-2}\right) I^{1} \sigma(T)
\end{aligned}
$$

where $\delta_{1}, \delta_{2}, \delta_{3}, \delta_{4}$ and $\delta$ are given by (2.2). This completes the proof.

\section{Existence results}

Let $C[0, T]$ denote the Banach space of all continuous real-valued functions defined on $[0, T]$ with the norm $\|u\|=\sup \{|u(t)|: t \in[0, T]\}$. For $t \in[0, T]$, define $u_{r}(t)=t^{r} u(t), r \geq 0$, and let $C_{r}[0, T]$ be the space of all functions $u_{r}$ such that $u \in C[0, T]$ which turns out to be a Banach space when endowed with the norm $\|u\|_{r}=\sup \left\{t^{r}|u(t)|: t \in[0, T]\right\}$.

Let us define an operator $\mathcal{Q}: C_{2-\alpha}[0, T] \rightarrow C_{2-\alpha}[0, T]$ as

$$
\begin{aligned}
(\mathcal{Q} u)(t)= & \int_{0}^{t} \frac{(t-s)^{\alpha-1}}{\Gamma(\alpha)} f(s, u(s)) d s \\
& +\left(\delta_{1} t^{\alpha-1}-\delta_{4} t^{\alpha-2}\right)\left[A \int_{0}^{\xi} \frac{(\xi-s)^{\alpha+\beta-1}}{\Gamma(\alpha+\beta)} f(s, u(s)) d s+a \int_{0}^{T} f(s, u(s)) d s\right] \\
& +\left(\delta_{2} t^{\alpha-1}+\delta_{3} t^{\alpha-2}\right)\left[B \int_{0}^{\eta} \frac{(\eta-s)^{\alpha+\beta-1}}{\Gamma(\alpha+\beta)} f(s, u(s)) d s\right. \\
& \left.+b \int_{0}^{T}(T-s) f(s, u(s)) d s\right] .
\end{aligned}
$$

Observe that problem (1.1) has solutions only if the operator $\mathcal{Q}$ has fixed points.

To establish the first existence result, we need the following fixed point theorem. 
Theorem 3.1 ([23]) Let E be a Banach space. Let $T: E \rightarrow E$ be a completely continuous operator, and let the set $V=\{x \in E \mid x=\mu T x, 0<\mu<1\}$ be bounded. Then the operator $T$ has a fixed point in $E$.

Theorem 3.2 Assume that there exists a constant $M>0$ such that $|f(t, u)| \leq M, \forall t \in$ $[0, T], u \in \mathbb{R}$. Then problem (1.1) has at least one solution in the space $C_{2-\alpha}[0, T]$.

Proof As a first step, we show that the operator $\mathcal{Q}$ is completely continuous. The continuity of $\mathcal{Q}$ follows from the continuity of $f$. Let $\mathcal{H}$ be a bounded set in $C_{2-\alpha}[0, T]$. Hence $\mathcal{H}$ is bounded on $C[0, T]$. Then, $\forall u \in \mathcal{H}, t \in[0, T]$, we have

$$
\begin{aligned}
& t^{2-\alpha} \mid(\mathcal{Q u}(t) \mid \\
& \leq M\left|t^{2-\alpha} \int_{0}^{t} \frac{(t-s)^{\alpha-1}}{\Gamma(\alpha)} d s+\left(\delta_{1} t-\delta_{4}\right)\right|\left[A \int_{0}^{\xi} \frac{(\xi-s)^{\alpha+\beta-1}}{\Gamma(\alpha+\beta)} d s+a \int_{0}^{T} d s\right] \\
& \quad+\left(\delta_{2} t+\delta_{3}\right)\left[B \int_{0}^{\eta} \frac{(\eta-s)^{\alpha+\beta-1}}{\Gamma(\alpha+\beta)} d s+b \int_{0}^{T}(T-s) d s\right] \\
& \leq M\left\{\frac{T^{2}}{\Gamma(\alpha+1)}+\left(\left|\delta_{1}\right|+\left|\delta_{4}\right|\right)\left(\frac{|A| \xi^{\alpha+\beta}}{\Gamma(\alpha+\beta+1)}+|a| T\right)\right. \\
&\left.\quad+\left(\left|\delta_{2}\right|+\left|\delta_{3}\right|\right)\left(\frac{|B| \eta^{\alpha+\beta}}{\Gamma(\alpha+\beta+1)}+\frac{|b| T^{2}}{2}\right)\right\} \\
&=L,
\end{aligned}
$$

which implies that $\|(\mathcal{Q} u)\|_{2-\alpha} \leq L$. Hence $\mathcal{Q H}$ is uniformly bounded. Also, for $t_{1}, t_{2} \in$ $[0, T], u \in \mathcal{H}$, we have

$$
\begin{aligned}
& \mid t_{1}^{2-\alpha}\left(\mathcal{Q u}\left(t_{1}\right)-t_{2}^{2-\alpha}(\mathcal{Q} u)\left(t_{2}\right) \mid\right. \\
& \leq M \mid \frac{1}{\Gamma(\alpha)} \int_{0}^{t_{1}}\left[t_{1}^{2-\alpha}\left(t_{1}-s\right)^{\alpha-1}-t_{2}^{2-\alpha}\left(t_{2}-s\right)^{\alpha-1}\right] d s-\frac{1}{\Gamma(\alpha)} \int_{t_{1}}^{t_{2}} t_{2}^{2-\alpha}\left(t_{2}-s\right)^{\alpha-1} d s \\
& \quad+\delta_{1}\left(t_{1}-t_{2}\right)\left[A \int_{0}^{\xi} \frac{(\xi-s)^{\alpha+\beta-1}}{\Gamma(\alpha+\beta)} d s+a \int_{0}^{T} d s\right] \\
& \quad+\delta_{2}\left(t_{1}-t_{2}\right)\left[B \int_{0}^{\eta} \frac{(\eta-s)^{\alpha+\beta-1}}{\Gamma(\alpha+\beta)} d s+b \int_{0}^{T}(T-s) d s\right] \mid \rightarrow 0 \quad \text { as } t_{1} \rightarrow t_{2} .
\end{aligned}
$$

Thus $t^{2-\alpha} \mathcal{Q H}$ and hence $\mathcal{Q H}$ is equicontinuous. So, by the Arzela-Ascoli theorem, $\mathcal{Q}$ is completely continuous. Next, we consider the set

$$
V=\left\{t^{2-\alpha} u \in \mathbb{R}: t^{2-\alpha} u=\mu t^{2-\alpha} \mathcal{Q} u, 0<\mu<1\right\},
$$

and show that $V$ is bounded. For $u \in V$, we have

$$
\left|t^{2-\alpha} u(t)\right|=\left|\mu t^{2-\alpha}(\mathcal{Q} u)(t)\right| \leq t^{2-\alpha}|\mathcal{Q} u(t)| \leq L
$$

This implies that the set $V$ is bounded independently of $\mu \in(0,1)$. Therefore, Theorem 3.1 applies and problem (1.1) has at least one solution on $[0, T]$. This completes the proof. 
Theorem 3.3 Assume that there exists a constant $K>0$ such that

$$
|f(t, u)-f(t, v)| \leq K|u-v|, \quad \forall t \in[0, T], u, v \in \mathbb{R}
$$

then problem (1.1) has a unique solution in $C_{2-\alpha}[0, T]$ if $K v<1$, where

$$
\begin{aligned}
v= & \left\{\frac{T^{2}}{\Gamma(\alpha+1)}+\frac{\left(\left|\delta_{1}\right|+\left|\delta_{4}\right|\right)\left(|A| \xi^{\alpha+\beta}+|a| T \Gamma(\alpha+\beta+1)\right)}{\Gamma(\alpha+\beta+1)}\right. \\
& \left.+\frac{\left(\left|\delta_{2}\right|+\left|\delta_{3}\right|\right)\left(2|B| \eta^{\alpha+\beta}+|b| T^{2} \Gamma(\alpha+\beta+1)\right)}{2 \Gamma(\alpha+\beta+1)}\right\} .
\end{aligned}
$$

Proof For every $t \in[0, T], u, v \in \mathbb{R}$, we have

$$
\begin{aligned}
t^{2-\alpha} \mid & (\mathcal{Q} u)(t)-(\mathcal{Q} v)(t) \mid \\
\leq & t^{2-\alpha} \int_{0}^{t} \frac{(t-s)^{\alpha-1}}{\Gamma(\alpha)}|f(s, u(s))-f(s, v(s))| d s \\
& +\left(\left|\delta_{1} t-\delta_{4}\right|\right)\left(|A| \int_{0}^{\xi} \frac{(\xi-s)^{\alpha+\beta-1}}{\Gamma(\alpha+\beta)}|f(s, u(s))-f(s, v(s))| d s\right. \\
& \left.+|a| \int_{0}^{T}|f(s, u(s))-f(s, v(s))| d s\right) \\
& +\left|\left(\delta_{2} t+\delta_{3}\right)\right|\left(|B| \int_{0}^{\eta} \frac{(\eta-s)^{\alpha+\beta-1}}{\Gamma(\alpha+\beta)}|f(s, u(s))-f(s, v(s))| d s\right. \\
& \left.+|b| \int_{0}^{T}(T-s)|f(s, u(s))-f(s, v(s))| d s\right) \\
\leq & K\left\{\frac{T^{2}}{\Gamma(\alpha+1)}+\frac{\left(\left|\delta_{1}\right|+\left|\delta_{4}\right|\right)\left(|A| \xi^{\alpha+\beta}+|a| T \Gamma(\alpha+\beta+1)\right)}{\Gamma(\alpha+\beta+1)}\right. \\
& \left.+\frac{\left(\left|\delta_{2}\right|+\left|\delta_{3}\right|\right)\left(2|B| \eta^{\alpha+\beta}+|b| T^{2} \Gamma(\alpha+\beta+1)\right)}{2 \Gamma(\alpha+\beta+1)}\right\}|u-v| .
\end{aligned}
$$

By the definition of $\|\cdot\|_{2-\alpha}$, we obtain

$$
\|(\mathcal{Q} u)(t)-(\mathcal{Q} v)(t)\|_{2-\alpha} \leq K v\|u-v\|_{2-\alpha} \leq\|u-v\|_{2-\alpha} .
$$

It follows that $\mathcal{Q}$ is a contraction. Hence, by the Banach contraction theorem, problem (1.1) has a unique solution in $C_{2-\alpha}[0, T]$. This completes the proof.

Our next existence result is based on Leray-Schauder nonlinear alternative [24].

Lemma 3.1 (Leray-Schauder's nonlinear alternative type) Let E be a Banach space, $M$ be a closed, convex subset of $E, U$ be an open subset of $C$ and $0 \in U$. Suppose that $F: \bar{U} \rightarrow C$ is a continuous, compact (that is, $F(U)$ is a relatively compact subset of $C$ ) map. Then either (i) F has a fixed point in $\bar{U}$ or (ii) there are $u \in \partial U$ and $\lambda \in(0,1)$ with $u=\lambda F(U)$.

Theorem 3.4 Let $f:[0,1] \times \mathbb{R} \rightarrow \mathbb{R}$ be a continuous function. Furthermore, assume that:

$\left(\mathrm{A}_{1}\right)$ There exist a function $p \in \mathcal{C}\left([0, T], \mathbb{R}^{+}\right)$and a nondecreasing function $\psi: \mathbb{R}^{+} \rightarrow \mathbb{R}^{+}$ such that $|f(t, u)| \leq p(t) \psi(\|u\|), \forall(t, u) \in[0, T] \times \mathbb{R}$; 
$\left(\mathrm{A}_{2}\right)$ There exists a constant $M>0$ such that

$$
\frac{M}{\psi(M) v\|p\|}>1 \text {, }
$$

where $v$ is given by (3.2).

Then boundary value problem (1.1) has at least one solution.

Proof First we shall show that the operator $\mathcal{Q}$ defined by (3.1) maps bounded sets into bounded ones in $C_{2-\alpha}([0, T], \mathbb{R})$. For $r>0$, let $\mathcal{H}_{r}=\left\{u \in C_{2-\alpha}[0, T]:\|u\|_{2-\alpha} \leq r\right\}$ be a bounded set in $C_{2-\alpha}([0, T], \mathbb{R})$. Then, for $u \in \mathcal{H}_{r}$, we have

$$
\begin{aligned}
t^{2-\alpha} \mid & (\mathcal{Q} u)(t) \mid \\
\leq & t^{2-\alpha} \int_{0}^{t} \frac{(t-s)^{\alpha-1}}{\Gamma(\alpha)} p(s) \psi\left(\|u\|_{2-\alpha}\right) d s \\
& +\left(\left|\delta_{1}\right|+\left|\delta_{4}\right|\right)\left(|A| \int_{0}^{\xi} \frac{(\xi-s)^{\alpha+\beta-1}}{\Gamma(\alpha+\beta)} p(s) \psi\left(\|u\|_{2-\alpha}\right) d s+|a| \int_{0}^{T} p(s) \psi\left(\|u\|_{2-\alpha}\right) d s\right) \\
& +\left(\left|\delta_{2}\right|+\left|\delta_{3}\right|\right)\left(|B| \int_{0}^{\eta} \frac{(\eta-s)^{\alpha+\beta-1}}{\Gamma(\alpha+\beta)} p(s) \psi\left(\|u\|_{2-\alpha}\right) d s\right. \\
& \left.+|b| \int_{0}^{T}(T-s) p(s) \psi\left(\|u\|_{2-\alpha}\right) d s\right) \\
\leq & \|p\| \psi(r)\left\{\frac{T^{2}}{\Gamma(\alpha+1)}+\left(\left|\delta_{1}\right|+\left|\delta_{4}\right|\right)\left(\frac{|A| \xi^{\alpha+\beta}}{\Gamma(\alpha+\beta+1)}+|a| T\right)\right. \\
& \left.+\left(\left|\delta_{2}\right|+\left|\delta_{3}\right|\right)\left(\frac{|B| \eta^{\alpha+\beta}}{\Gamma(\alpha+\beta+1)}+\frac{|b| T^{2}}{2}\right)\right\} \\
\leq & \|p\| \psi(r) v,
\end{aligned}
$$

where $v$ is given by (3.2).

Next, we shall show that the operator $\mathcal{Q}$ maps bounded sets into equicontinuous sets. Let $t_{1}, t_{2} \in[0, T]$ with $t_{1}<t_{2}$ and $u \in \mathcal{H}_{r}$. Then we have

$$
\begin{aligned}
& \left|t_{1}^{2-\alpha}(\mathcal{Q} u)\left(t_{1}\right)-t_{2}^{2-\alpha}(\mathcal{Q} u)\left(t_{2}\right)\right| \\
& \leq \mid \frac{1}{\Gamma(\alpha)} \int_{0}^{t_{1}}\left[t_{1}^{2-\alpha}\left(t_{1}-s\right)^{\alpha-1}-t_{2}^{2-\alpha}\left(t_{2}-s\right)^{\alpha-1}\right] p(s) \psi(r) d s \\
& \quad-\frac{1}{\Gamma(\alpha)} \int_{t_{1}}^{t_{2}} t_{2}^{2-\alpha}\left(t_{2}-s\right)^{\alpha-1} p(s) \psi(r) d s \\
& \quad+\delta_{1}\left(t_{1}-t_{2}\right)\left(A \int_{0}^{\xi} \frac{(\xi-s)^{\alpha+\beta-1}}{\Gamma(\alpha+\beta)} p(s) \psi(r) d s+a \int_{0}^{T} p(s) \psi(r) d s\right) \\
& \quad+\delta_{2}\left(t_{1}-t_{2}\right)\left(B \int_{0}^{\eta} \frac{(\eta-s)^{\alpha+\beta-1}}{\Gamma(\alpha+\beta)} p(s) \psi(r) d s\right. \\
& \left.\quad+b \int_{0}^{T}(T-s) p(s) \psi(r) d s\right) \mid \\
& \leq\|p\| \psi(r) \mid \frac{1}{\Gamma(\alpha)} \int_{0}^{t_{1}}\left[t_{1}^{2-\alpha}\left(t_{1}-s\right)^{\alpha-1}-t_{2}^{2-\alpha}\left(t_{2}-s\right)^{\alpha-1}\right] d s
\end{aligned}
$$




$$
\begin{aligned}
& -\frac{1}{\Gamma(\alpha)} \int_{t_{1}}^{t_{2}} t_{2}^{2-\alpha}\left(t_{2}-s\right)^{\alpha-1} d s+\delta_{1}\left(t_{1}-t_{2}\right)\left(A \int_{0}^{\xi} \frac{(\xi-s)^{\alpha+\beta-1}}{\Gamma(\alpha+\beta)} d s\right. \\
& \left.+a \int_{0}^{T} p(s) \psi(r) d s\right)+\delta_{2}\left(t_{1}-t_{2}\right)\left(B \int_{0}^{\eta} \frac{(\eta-s)^{\alpha+\beta-1}}{\Gamma(\alpha+\beta)} d s\right. \\
& \left.+b \int_{0}^{T}(T-s) d s\right) \mid
\end{aligned}
$$

which tends to zero independently of $u \in \mathcal{H}_{r}$ as $t_{1} \rightarrow t_{2}$. Thus $\mathcal{Q}$ is completely continuous. Now let $u$ be a solution of problem (1.1), then for $t \in[0, T]$ and $\lambda \in(0,1)$, we have

$$
\begin{aligned}
t^{2-\alpha}|u(t)| \leq & \psi\left(\|u\|_{2-\alpha}\right)\|p\|\left\{\frac{T^{2}}{\Gamma(\alpha+1)}+\left(\left|\delta_{1}\right|+\left|\delta_{4}\right|\right)\left(\frac{|A| \xi^{\alpha+\beta}}{\Gamma(\alpha+\beta+1)}+|a| T\right)\right. \\
& \left.+\left(\left|\delta_{2}\right|+\left|\delta_{3}\right|\right)\left(\frac{|B| \eta^{\alpha+\beta}}{\Gamma(\alpha+\beta+1)}+\frac{|b| T^{2}}{2}\right)\right\},
\end{aligned}
$$

which can be rewritten as

$$
\begin{aligned}
& t^{2-\alpha}|u(t)|\left[\psi ( \| u \| _ { 2 - \alpha } ) \| p \| \left\{\frac{T^{2}}{\Gamma(\alpha+1)}+\left(\left|\delta_{1}\right|+\left|\delta_{4}\right|\right)\left(\frac{|A| \xi^{\alpha+\beta}}{\Gamma(\alpha+\beta+1)}+|a| T\right)\right.\right. \\
& \left.\left.+\left(\left|\delta_{2}\right|+\left|\delta_{3}\right|\right)\left(\frac{|B| \eta^{\alpha+\beta}}{\Gamma(\alpha+\beta+1)}+\frac{|b| T^{2}}{2}\right)\right\}\right]^{-1} \leq 1
\end{aligned}
$$

By assumption $\left(\mathrm{A}_{2}\right)$, there exists $M$ such that $t^{2-\alpha}|u(t)| \neq M$. Let us set

$$
\mathcal{H}_{M}=\left\{u \in C_{2-\alpha}[0, T]: t^{2-\alpha}|u(t)|<M+1\right\}
$$

Note that the operator $\mathcal{Q}: \overline{\mathcal{H}}_{M} \rightarrow C_{2-\alpha}[0, T]$ is completely continuous and by the definition of $\mathcal{H}_{M}$, there is no $u \in \partial \mathcal{H}_{M}$ such that $u=\lambda \mathcal{Q}(u)$ for some $\lambda \in(0,1)$. In consequence, by Lemma 3.1, we conclude that $\mathcal{Q}$ has at least one fixed point $u \in \overline{\mathcal{H}}_{M}$, which is a solution of problem (1.1).

\section{Examples}

Example 4.1 Consider the following fractional integral boundary value problem:

$$
\left\{\begin{array}{l}
D^{\alpha} u(t)=\frac{\sin u(t)+\cos u(t)}{5+\cos ^{2} u(t)}, \quad t \in[0,1] \\
D^{0.5} x\left(0^{+}\right)-D^{0.5} x\left(1^{-}\right)=I^{7 / 4} x(1 / 2) \\
D^{-0.5} x\left(0^{+}\right)-D^{-0.5} x\left(1^{-}\right)=I^{7 / 4} x(3 / 4)
\end{array}\right.
$$

Since

$$
|f(t, u)|=\left|\frac{\sin u(t)+\cos u(t)}{5+\cos ^{2} u(t)}\right| \leq \frac{2}{5},
$$

therefore, Theorem 3.2 applies and problem (4.1) has at least one solution on $[0,1]$.

Example 4.2 Consider the problem

$$
\left\{\begin{array}{l}
D^{\frac{3}{2}} x(t)=K\left(\cos t+\tan ^{-1} x(t)\right), \quad t \in[0,1] \\
D^{0.5} x\left(0^{+}\right)-D^{0.5} x\left(1^{-}\right)=I^{7 / 4} x(1 / 2) \\
D^{-0.5} x\left(0^{+}\right)-D^{-0.5} x\left(1^{-}\right)=I^{7 / 4} x(3 / 4) .
\end{array}\right.
$$


Here $\alpha=3 / 2, T=1, a=b=1, \xi=1 / 2, \eta=3 / 4, \beta=7 / 4, A=B=1$. Clearly,

$$
|f(t, u)-f(t, v)| \leq K\left|\tan ^{-1} u(t)-\tan ^{-1} v(t)\right| \leq K|u-v|
$$

$v \simeq 4.315066$ ( $v$ is given by (3.2)) and in consequence, $K<0.231746$. Thus, all the assumptions of Theorem 3.3 are satisfied. Therefore, by the conclusion of Theorem 3.3, there exists a unique solution for problem (4.2).

\section{Competing interests}

The authors declare that they have no competing interests.

\section{Authors' contributions}

Each of the authors, BA, AA, AAS and RPA contributed to each part of this work equally and read and approved the final version of the manuscript.

\section{Author details}

'Department of Mathematics, Faculty of Science, King Abdulaziz University, P.O. Box 80203, Jeddah, 21589, Saudi Arabia.

${ }^{2}$ Department of Mathematics, Texas A\&M University, Kingsville, TX 78363-8202, USA.

\section{Acknowledgements}

This research was partially supported by the Deanship of Scientific Research (DSR), King Abdulaziz University, Jeddah, Saudi Arabia.

Received: 29 July 2013 Accepted: 25 November 2013 Published: 13 Dec 2013

\section{References}

1. Podlubny, I: Fractional Differential Equations. Academic Press, San Diego (1999)

2. Kilbas, AA, Srivastava, HM, Trujillo, Jj: Theory and Applications of Fractional Differential Equations. North-Holland Mathematics Studies, vol. 204. Elsevier, Amsterdam (2006)

3. Sabatier, J, Agrawal, OP, Machado, JAT (eds.): Advances in Fractional Calculus: Theoretical Developments and Applications in Physics and Engineering. Springer, Dordrecht (2007)

4. Baleanu, D, Diethelm, K, Scalas, E, Trujillo, Jj: Fractional Calculus Models and Numerical Methods. Series on Complexity, Nonlinearity and Chaos. World Scientific, Boston (2012)

5. Ahmad, B, Nieto, JJ: Riemann-Liouville fractional integro-differential equations with fractional nonlocal integral boundary conditions. Bound. Value Probl. 2011, 36 (2011)

6. Ahmad, B, Ntouyas, SK: A four-point nonlocal integral boundary value problem for fractional differential equations of arbitrary order. Electron. J. Qual. Theory Differ. Equ. 2011, 22 (2011)

7. Zhang, X, Wang, L: Nontrivial solutions for a class of fractional differential equations with integral boundary conditions and a parameter in a Banach space with lattice Abstr. Appl. Anal. 2012, Article ID 391609 (2012)

8. Guezane-Lakoud, A, Khaldi, R: Solvability of a fractional boundary value problem with fractional integral condition. Nonlinear Anal. 75, 2692-2700 (2012)

9. Sudsutad, W, Tariboon, J: Boundary value problems for fractional differential equations with three-point fractional integral boundary conditions. Adv. Differ. Equ. 2012, 93 (2012)

10. Ahmad, B, Ntouyas, SK, Alsaedi, A: On fractional differential inclusions with anti-periodic type integral boundary conditions. Bound. Value Probl. 2013, 82 (2013)

11. Fu, D, Ding, W: Existence of positive solutions of third-order boundary value problems with integral boundary conditions in Banach spaces. Adv. Differ. Equ. 2013, 65 (2013)

12. Vong, S: Positive solutions of singular fractional differential equations with integral boundary conditions. Math. Comput. Model. 57, 1053-1059 (2013)

13. Alsaedi, A, Ntouyas, SK, Ahmad, B: Existence results for Langevin fractional differential inclusions involving two fractional orders with four-point multiterm fractional integral boundary conditions. Abstr. Appl. Anal. 2013, Article ID $869837(2013)$

14. Baleanu, D, Rezapour, S, Mohammadi, H: Some existence results on nonlinear fractional differential equations. Philos. Trans. R. Soc. A, Math. Phys. Eng. Sci. 371(1990), 20120144 (2013)

15. Baleanu, D, Mustafa, OG, O'Regan, D: A uniqueness criterion for fractional differential equations with Caputo derivative. Nonlinear Dyn. 71, 635-640 (2013)

16. Darzi, R, Mohammadzadeh, B, Neamaty, A, Baleanu, D: Lower and upper solutions method for positive solutions of fractional boundary value problems. Abstr. Appl. Anal. 2013, Article ID 847184 (2013)

17. Zhou, WX, Chu, YD, Baleanu, D: Uniqueness and existence of positive solutions for a multi-point boundary value problem of singular fractional differential equations. Adv. Differ. Equ. 2013, 114 (2013)

18. Wang, G, Ahmad, B, Zhang, L, Agarwal, RP: Nonlinear fractional integro-differential equations on unbounded domains in a Banach space. J. Comput. Appl. Math. 249, 51-56 (2013)

19. Ahmad, B, Ntouyas, SK, Alsaedi, A: A study of nonlinear fractional differential equations of arbitrary order with Riemann-Liouville type multistrip boundary conditions. Math. Probl. Eng. 2013, Article ID 320415 (2013)

20. Liu, Y, He, T, Shi, H: Three positive solutions of Sturm-Liouville boundary value problems for fractional differential equations. Differ. Equ. Appl. 5, 127-152 (2013) 
21. Eldar, V, Turab, A, Maksym, l: Fractional operators approach and fractional boundary conditions. In: Zhurbenko, V (ed.) Electromagnetic Waves (2011). ISBN:978-953-307-304-0. InTech, doi:10.5772/16300

22. Ahmad, B, Nieto, JJ: Riemann-Liouville fractional differential equations with fractional boundary conditions. Fixed Point Theory 13, 329-336 (2012)

23. Smart, DR: Fixed Point Theorems. Cambridge University Press, Cambridge (1980)

24. Granas, A, Dugundji, J: Fixed Point Theory. Springer, New York (2005)

10.1186/1687-2770-2013-274

Cite this article as: Ahmad et al.: A study of Riemann-Liouville fractional nonlocal integral boundary value problems. Boundary Value Problems 2013, 2013:274

Submit your manuscript to a SpringerOpen ${ }^{\circ}$ journal and benefit from:

- Convenient online submission

- Rigorous peer review

Immediate publication on acceptance

Open access: articles freely available online

- High visibility within the field

- Retaining the copyright to your article

Submit your next manuscript at $>$ springeropen.com 